\title{
Corrigendum
}

\section{Clinical outcome, healthcare cost and length of hospital stay among patients with bloodstream infections and acute leukemia in a cancer center in Eastern India - CORRIGENDUM}

In the article by Muennichow et $\mathrm{al}^{1}$ the degree certification of author Arpita Bhattacharyya was incorrect on the original published version. The correct degree certification is MRCP. The authors apologize for the error.

\section{Reference}

1. Muennichow CE, Goel G, Bhattacharyya A, et al. Clinical Outcome, Healthcare Cost and Length of Hospital Stay Among Patients With Bloodstream Infections and Acute Leukemia in a Cancer Center in Eastern India. Infection Control Hosp Epidemiol 2018;39:1013-1014. 